Ann. Génét. Sél. anim., 1974, 6 (3), 381-385.

\title{
DIFFÉRENCE ENTRE SEXES POUR LE POIDS DU POUSSIN EXPRIMÉ EN POURCENTAGE DU POIDS DE L'CEUF : RELATIONS AVEC LA CROISSANCE ULTÉRIEURE
}

\author{
J. SILBER et P. MÉRAT \\ Laboratoire de Génétique factorielle, \\ Centre national de Recherches zootechniques, I. N. R. A., \\ 78350 Jouy en Josas \\ RÉSUMÉ
}

Le poìds des poussins mâles à l'éclosion, exprimé en pourcentağe du poids de l'œuf, est trouvé très significativement plus élevé que celui des poussins femelles. Par contre, le poids des œufs donnant des femelles écloses est significativement plus élevé que celui des œufs donnant des mâles.

A poids du poussin fixé, chez les $\widehat{\delta} \hat{\sigma}$, il ne semble pas y avoir de relation entre le poids du poussin exprimé en pourcentage du poids de l'œuf, et la croissance ultérieure.

\section{INTRODUCTION}

Le but de ce travail est de voir s'il existe des différences entre sexes pour le poids du poussin exprimé en pourcentage du poids de l'œuf.

Dans un deuxième temps, nous avons cherché s'il existait des liaisons entre ce pourcentage et la croissance ultérieure de l'animal.

JUL, (I923) et JULL, et QUINN (I925) trouvent qu'il n'y a pas de différence significative entre sexes dans le poids du poussin à la naissance. JUL, et HEYwaNG (r930) exprimant le poids du poussin comme nous l'avons fait ne trouvent pas non plus de différence entre sexes. Par contre, MuNRo et Kosrn (I940) en comparant des poussins issus d'une même mère trouvent que les $\widehat{\partial} \hat{\delta}$ sont plus lourds que les 우, et que le poids du poussin exprimé en pourcentage du poids de l'œuf est plus important pour les $\widehat{\sigma} \delta$. 


\section{MATÉRIEL, ET MÉTHODES}

Notre troupeau expérimental est mis à l'âge de I I mois en parquets de reproduction. Les parquets, individuels, comprennent I coq pour ro poules. Après une période d'adaptation, les œufs sont ramassés quotidiennement pendant 5 semaines consécutives. La ponte est contrôlée en nid trappe, chaque œuf pesé, et à la fin de chaque semaine les œufs sont mis en incubation. Les poussins sont sortis de l'incubateur au $22^{\mathrm{e}}$ jour et sexés et pesés aussitôt après.

La différence de poids, exprimée en pourcentage du poids de l'œuf, des poussins $\sigma^{\star} \sigma^{\star}$ et 우우 issus d'une même mère a été testée par la méthode des couples. Chaque couple était constitué pour une mère donnée par le rapport du poids moyen des poussins ô ô au poids moyen de l'œuf et de même pour les poussins ợ. Nous avons utilisé le même test pour mettre en évidence la différence du poids des cufs ayant donné un poussin $\delta$ et de ceux ayant donné un poussin ; ainsi que la différence de poids à la naissance des poussins des deux sexes.

\section{RÉSULTATS ET DISCUSSION}

I. - Comparaison entre sexes pour le poids du poussin à la naissance, le poids de l'cuf dont il est issu, le poids du poussin exprimé en pourcentage du poids de l'cuf

Les résultats sont donnés dans le tableau I.

Nous observons un poids des poussins $\hat{\sigma}$ exprimé en pourcentage du poids de l'œuf très significativement plus élevé que le poids des poussins $q$.

\section{TABLEAU I}

Comparaison entre sexes du poids du poussin à la naissance, du poids de l'ceuf dont il est issu, et du poids du poussin,

exprimé en pourcentage du poids de l'ceuf pour des animaux issus d'une même mère

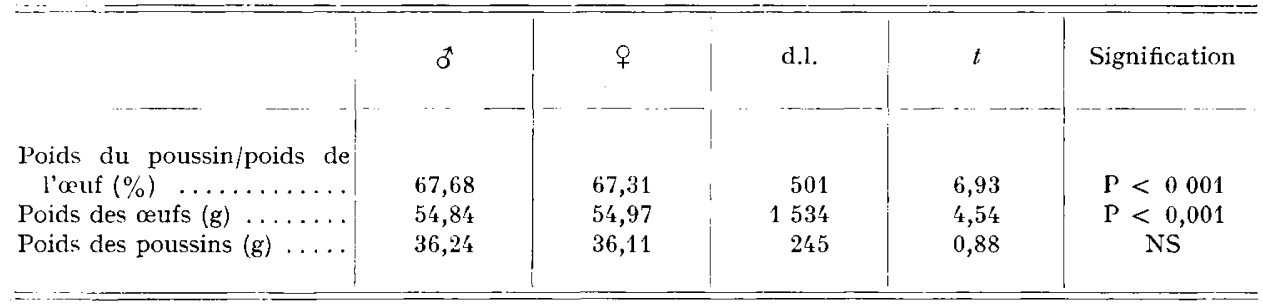

Le poids des œufs donnant des $q \nmid$ est très significativement plus élevé que celui des œufs donnant des ổ.

Bien que les poussins $\hat{\delta} \hat{o}$ à la naissance paraissent être très légèrement plus lourds en valeur absolue que leurs sœurs, la différence n'est pas significative.

Nos résultats relatifs au poids des poussins exprimé en pourcentage du poids de l'œuf vont dans le même sens que ceux de MunRo et Kosin (I940). Par contre ces derniers ne trouvent pas de différence pour le poids des œufs donnant des poussins $\widehat{\delta} \hat{\delta}$ et $ㅇ ㅜ$ mais trouvent les poussins $\hat{\delta} \widehat{\partial}$ significativement plus lourds. Ces auteurs utilisent eux aussi le test des couples. Leur résultat peut s'expliquer par le fait que les poussins $q$ 우 éclosent relativement plus tôt que les poussins $\widehat{o} \hat{\sigma}$ (ICHNOE et al., 
I967). Les premiers, au moment de la pesée, ont donc pu subir plus fortement la perte de poids qui survient après la naissance. Nos propres poussins ne sont pesés que 24 heures après l'éclosion et la différence du poids des poussins $\delta^{\star} \sigma^{\wedge}$ et $+{ }^{\circ}+n^{\prime}$ 'est plus significative.

Comment interpréter la différence observée dans le poids des œufs donnant

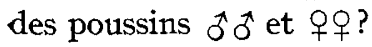

Pour une même poule, la moyenne du poids des œufs donnant des poussins 우우 est de $0,13 \mathrm{~g}$ plus importante que celle donnant des poussins $\widehat{b} \widehat{b}$.

On peut tenter d'expliquer cette différence par l'hypothèse suivante : on sait que les œufs de faible poids ont une mortalité embryonnaire légèrement plus élevée (parfois aussi les très gros œufs). On peut alors penser que les embryons 우우 sont légèrement plus sensibles aux conditions moins favorables apportées par ces petits œufs et présentent une mortalité un peu plus élevée que les $\widehat{0} \delta^{\star}$, de sorte que les embryons femelles ayant survécu proviendront en proportion plus faible d'œufs de petite taille, d'où la supériorité du poids moyen des œufs leur donnant naissance. Le caractère souvent plus fragile du sexe hétérogamétique serait en faveur de cette hypothèse. Toutefois, on n'a jamais prouvé que la mortalité embryonnaire d'une façon générale soit plus élevée pour les zygotes + chez la poule, hormis le cas de gènes létaux liés au sexe. D'autre part, nous savons que le " sex ratio " secondaire généralement observé est très voisin de la proportion $I / I$ chez le Poulet. Notre hypothèse présupposerait un " sex ratio " primaire légèrement supérieur en faveur des 우오. En fait, le phénomène que nous observons ici s'accorderait avec un écart du "sex ratio " secondaire à la proportion $\mathrm{I} / \mathrm{I}$ relativement faible, et qui pourrait passer inaperçu avec les tests statistiques usuels.

Inversement, un léger excès de zygotes 우 au stade du " sex ratio " primaire n'est pas totalement exclu a priori. Nous savons que chez l'Homme (Revue bibliographique de STEVENSON et BoBRow, 1967) il y a une légère prépondérance de mâles à la naissance et que cet excès de $\sigma^{\hat{t}}$ est plus sensible encore si l'on observe les morts embryonnaires. Comme chez les humains, des morts embryonnaires, probablement précoces, pourraient être dûs, chez la Poule, à la présence de gènes létaux portés par les chromosomes sexuels.

Le seul résultat connu chez la Poule est celui de FechHeIMER et al. (I970) qui trouvent un sex ratio de 49,45 p. Ioo (non significativement différent de $50 \mathrm{p}$. IOo) à I6 heures d'incubation sur 918 embryons. Ce résultat n'est donc pas concluant en ce qui concerne l'hypothèse émise ici. Nous devons donc la considérer avec prudence et tester nos résultats sur d'autres populations, en déterminant dans la mesure du possible le sexe des embryons morts.

Une autre question qui reste posée, en fin de compte, est celle de savoir comment l'embryon $\delta^{\dagger}$ en partant des réserves nutritives du même ordre que l'embryon $q$, les utilise d'une meilleure façon.

\section{2. - Corrélation entre le poids du poussin}

exprimé en pourcentage du poids de l'auf et le poids ultérieur de l'animal

Nous nous sommes demandés, étant donnée la variation qui existe dans le poids du poussin en pourcentage du poids de l'œuf des enfants du même sexe, d'une mère à l'autre, si celui-ci pouvait avoir une relation avec la croissance ultérieure. 
Les résultats sont donnés dans le tableau 2 . Nous nous sommes limités au sexe 0 . Les calculs ont été faits à partir des résultats de quatre années (I969 à 1972 inclus).

\section{TABLEAU 2}

Corrélations entre le poids du poussin o exprimé en pourcentage du poids de l'ceuf et la croissance ultérieure

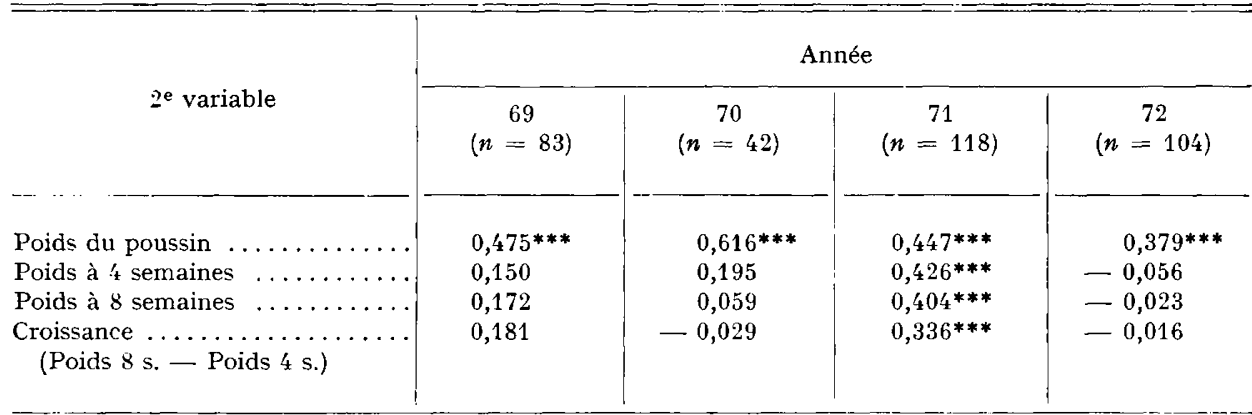

* Significatif à 5 p. 100.

** Significatif à 1 p. 100.

*** Significatif à 1 p. 1000 .

Le poids du poussin en pourcentage du poids de l'œuf est toujours très fortement lié au poids absolu du poussin. Les liaisons entre la première variable et les poids à 4 semaines, à 8 semaines, et la croissance de 4 à 8 semaines paraissent varier d'un troupeau à 1'autre et sont significativement différentes entre elles.

Dans le tableau 3 nous donnons les mêmes corrélations à poids de poussin fixé.

TABIEAU 3

Corrélations partielles entre le poids du poussin $\delta$ exprimé en pourcentage du poids de l'œuf

et la croissance ultérieure, à poids de poussin fixé

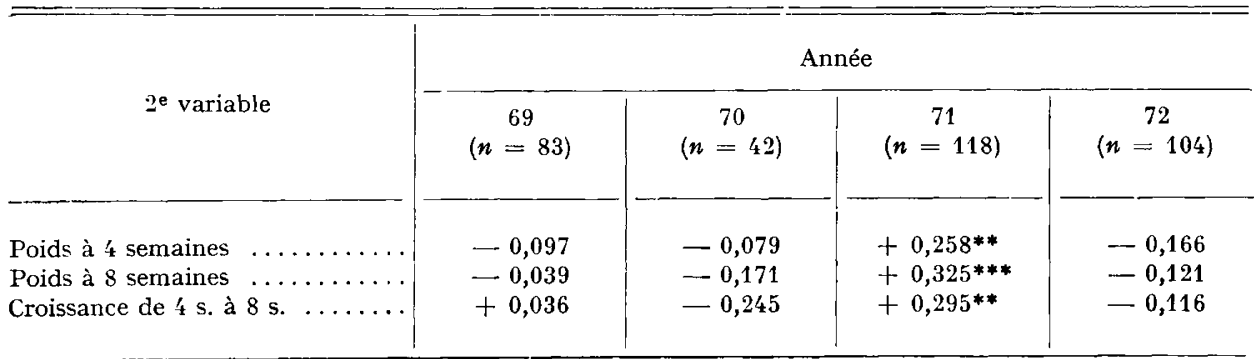

* Significatif à 5 p. 100.

** Significatif à 1 p. 100 .

*** Significatif à 1 p. 1000. 
Nous voyons que pour un poids de poussin fixé, la liaison entre le poids du poussin en pourcentage du poids de l'œuf et le poids ultérieur varie encore de façon considérable d'un troupeau à l'autre.

WASHBURN et GUILL (I973) ont étudié la croissance embryonnaire définie comme le poids de l'embryon à ro jours exprimé en pourcentage du poids de l'œuf et l'efficacité alimentaire du poussin âgé de 5 à 7 semaines et de 7 à 9 semaines, ils ne trouvent pas de liaison significative entre ces caractères. Ils n'observent pas non plus de liaison entre le poids du poussin exprimé tel que nous l'avons fait et l'efficacité alimentaire de 5 à 7 semaines $(+0,05)$ et de 7 à 9 semaines $(-0,02)$; la corrélation avec la croissance de 5 à 7 semaines est de + o, I 4 et de 7 à 9 semaines de $+0,12$. Ces auteurs concluent que les gènes responsables des différences de croissance et d'efficacité ne sont pas actifs à l'état embryonnaire.

A la lumière de nos résultats nous pensons, étant donnée la variabilité des réponses d'un troupeau à l'autre, qu'il serait difficile d'utiliser des différences entre familles pour le poids du poussin exprimé en pourcentage du poids de l'œuf.

Reçu pour publication en septembre 1974.

\section{SUMMARY}

\section{DIFFERENCES BETWEEN SEXES FOR THE RATIO OF CHICK WEIGHT TO EGG WEIGHT : RELATIONSHIP WITH POST-EMBRYONIC GROWTH}

The weight of hatched male chicks, expressed in percent of egg weight, is found very significantly higher than that of female chicks. Conversely, the weight of eggs giving hatched females is significantly higher than that of eggs giving males.

At fixed chick weight, there does not seem to be any relation between chick weight expressed in percent of egg weight, and further growth.

\section{RÉFÉRENCES BIBLIOGRAPHIQUES}

Fechheimer N. S., Lodge J. R., Miller R. C., I97o. Sex proportion of domestic chicken at i6 hours of incubation. J. Reprod. Fert., 23, 365-367.

Ichinoe K., Ohta E., Makabe T., Ichige T., Suzuki S., 1967. The relation between hatching time and sex ratio in chicks. Jap. poult. Sci., 4 20r-206.

Jull M. A., I923. Differential sex growth curves in Barred Plymouth Rock chicks. Sci. Agric., 4, 58-65.

Jull M. A., HeYwang B. W., 1930. Yolk assimilation during the embryonic development of the chick. Poult. Sci., 9, 393-404.

Jull M. A., Quins J. F., r925. The relationship between the weight of eggs and weight of chicks according to sex. Jour. A gric. Res., 31, 223-226.

Munro S. S., Kosin I. L., I940. The existence of a sex difference in the weight of day-old chicks, with further data on the egg weight-chick weight relationship. Sci. Agr., 20, 586-591.

Stevenson A. C., Bobrow M., I967. Determinants of sex proportion in man, with consideration of the evidence concerning a contribution from $\mathrm{X}$-linked mutations to intra-uterine death. J. Medical Genetics, 4, rg0-221.

Washburn K. W., Guill R. A., 1974. Relationship of embryo weight as a percent of egg weight to efficiency of feed utilization in the hatched chick. Poult. Sci., 58, 766-769. 